\title{
Development of Direct Water Purification System
}

\author{
Doo-Sung Baik ${ }^{1}$ \\ ${ }^{1}$ Dept. of Mechanical Engineering, Daejin University, Korea \\ ${ }^{1}$ dsbaik@daejin.ac.kr
}

\begin{abstract}
After the mid-1980s, a membrane-based drinking water supply device was first studied in Korea, and recently, a reverse osmosis pressure drinking supply device and UF hollow fiber membrane drinking water supply device are the main types. The cooling method of water purifier is largely divided into two. There is a direct cooling method that cools the water by installing an evaporator inside the water purifier cold water tank, and the indirect cooling method that cools the water inside the reservoir by cooling the entire cold water container by placing the evaporator outside the cold water container. However, since water is always stored in the cold water tank, it is necessary to clean the water bottle due to bacteria growth. In addition, the cooling water tank must operate the cooling system frequently regardless of the use of drinking water to maintain a constant temperature at all times, and unnecessary power required for standby is consumed. On the other hand, the direct water purifier cooling system has a lower continuous discharge rate compared to the storage type. In this study, by improving the existing instantaneous cooling system, the cooling coil is directly in contact with the cold water pipe so that heat is directly transferred by the heat conduction method that occurs between the cooling coil and the cold water pipe, thereby contributing to the energy saving.
\end{abstract}

Keywords: Water purifier, Cooling system, Refrigerants, Filter, Cooling coil

\section{Introduction}

The water purifier industry is an industry that requires advanced water treatment technology through the process of separating and filtering pollutants. Until the mid-1990s, the core parts of water purifiers depended on the United States and Japan, but many domestic companies recently produced finished products as well as water purifier parts, and the water purifier market is overheating. Water purifiers are widely applied in the fields of drinking water and food and beverages, and particularly interest in health and well-being due to increased income is increasing the preference for premium products with multi-function and high performance. In order to satisfy the needs of consumers, it is necessary to develop a multifunctional highquality water purifier such as a small ice water purifier, carbonated water purifier, or coffee water purifier [1]. In order to satisfy the needs of consumers, it is necessary to develop a multifunctional high-quality water purifier such as a small ice water purifier, carbonated water purifier, or coffee water purifier [1]. Water purifiers are recognized as home appliances that are essential to health in each household, and domestic water purifier companies are estimated to be about 150. Competition to enter the market is intensifying, and in 1998, water purifier companies started renting water purifiers to secure the market, allowing consumers to

Article history:

Received (September 8, 2020), Review Result (October 14, 2020), Accepted (December 6, 2020) 
use them conveniently at a very low cost, changing their perception of the use of water purifiers in the past. On the one hand, consumers' awareness of water purifiers has increased from time to time due to anxiety about water quality and the growth of living environment [2]. Domestic water purifiers, including Coway and Cheongho Nice, have also entered the water purifier business, and the domestic water purifier industry is considered to be full. While Chungho Nice and Coway are releasing expensive products, a lot of low-end products are being sold on the market. Currently, the water purifier business is mostly supplied to the market through rental services. Due to its characteristics, reliability with consumers is important, and due to concerns about water quality, consumers are demanding water purifiers with various functions [3]. Therefore, it is predicted that in the water purifier industry, intelligentization of material parts such as filters and valves and electronic parts is required. In addition, it is demanding a water purifier that is compact and emphasizes the beauty of its appearance, and recently, some products incorporating AI have been released. Meanwhile, as competition for domestic water purifiers intensifies, some companies are turning their sales strategies to overseas markets with poor water quality [4].

The core technology of water purifier is filter and cooling system. The cooling method applied to the water purifier uses an inverter compressor and a semiconductor cooling method using a thermoelectric element. The inverter compressor method is a method that uses the heat of vaporization and liquefaction of the refrigerant using a compressor and an evaporator, and has high cooling efficiency and is a method generally applied to refrigerators because it is possible to adjust the fine temperature without being affected by the ambient temperature. The semiconductor cooling method using a thermoelectric element connects different types of metals or semiconductors to flow current. The semiconductor cooling method using a thermoelectric element connects different types of metals or semiconductors to flow current.

The semiconductor cooling method using a thermoelectric element connects different types of metals or semiconductors to flow current.

Semiconductor cooling method using a thermoelectric element is connected to flow a current to the different kind of metal or semiconductor.

Semiconductor cooling method using a thermoelectric element is connected to flow a current to the different kind of metal or semiconductor.

It is a method that uses the phenomenon of high temperature on one side and low temperature on the other, and it circulates and cools the cold air of the contact point that has reached low temperature. Compared to the compressor method, noise and vibration are less, but the cooling speed is slow and it is relatively affected by the ambient temperature. However, it is suitable for water purifiers with reservoirs [5].

Water purifiers are classified as liquid filters for product classification, and are devices manufactured to meet the standards of drinking water quality through physical, chemical or biological processes. The current water purifier market is being developed and sold from large to small sizes for home use according to technological development. According to the raw water supply method, it can be divided into storage type water purifier and direct water type water purifier. On the other hand, according to the function of using the water purifier, it can be classified into cold and hot water purifier, cold water purifier, and general water purifier. If the water purifier is classified according to its type, it can be divided into a direct water type, which is a method of directly connecting the water purifier to a faucet, and a storage type, which stores water purified by passing raw water through a filter. Storage type water purifiers have a reservoir that receives raw water and stores purified water through a filter. This water purifier has a cold water container and a hot water container according to the 
optional function of the water purifier. Since it stores a certain amount at the required temperature, a lot of hot and cold water.

Storage type water purifiers have a reservoir that receives raw water and stores purified water through a filter. This water purifier has a cold water container and a hot water container according to the optional function of the water purifier. Since it stores a certain amount at the required temperature, a lot of hot and cold water

Storage-type water purifier there is a reservoir to store the purified water through the filter when supplied the raw integer tub and the water passes through the hot water tank in accordance with the water purifier optional features many hot and cold water because they keep a certain amount to the required temperature

It has the advantage of being able to drink it when convenient. On the other hand, since there is a reservoir, it is highly likely to be contaminated by bacterial growth, so periodic management is required. Therefore, a separate system is being developed to prevent the growth of bacteria. Recently, a water purifier was developed that periodically cleans the pipeline by converting the state of hot water into steam with a steam sterilization function. Since the direct water purifier can receive the raw water required and instantaneously provide it as drinking water, it is supplied as drinking water after receiving water through a connected water supply without a storage reservoir, purifying through a filter. Since the direct water purifier can receive the raw water required and instantaneously provide it as drinking water, it is supplied as drinking water after receiving water through a connected water supply without a storage reservoir, purifying through a filter.

Straight male purifier is adapted to supply the required raw water supplied directly to receive a constant after beverage through the filter when supplied with water to be connected without a reservoir to store it is possible to provide a momentarily drinking water.

Therefore, the direct water purifier has a low risk of bacterial propagation and does not need to keep cold water and hot water constant, thereby reducing standby power and saving electricity bills. However, since purified water is not stored as much as the capacity of the reservoir, there is a disadvantage in that the flow rate of cold and hot water is insufficient [6].

Looking at the filter technology of the water purifier, the filter applied to the water purifier consists of 1 to 4 steps, and is sequentially composed of a pre-carbon filter, a hollow fiber membrane filter or a reverse osmosis filter, and a post carbon filter from the first-stage sediment filter. Of these, three steps are important. In the case of a hollow fiber membrane filter, the pore size is fine in the range of 0.001 to $0.1 \mu \mathrm{m}$, so it is possible to almost completely remove impurities such as suspended substances, relatively large common bacteria, E. coli, and chemical contaminants, but heavy metals, bacteria and virus removal is impossible. It has a selective filtration function to pass minerals, and it is suitable for tap water purification because it is advantageous in durability or maintenance. On the other hand, the reverse osmosis filter uses a reverse osmosis phenomenon in which water moves toward a concentrated solution as opposed to the osmosis phenomenon when a high pressure is applied to a solution with a high concentration. On the other hand, the reverse osmosis filter uses a reverse osmosis phenomenon in which water moves toward a concentrated solution as opposed to the osmosis phenomenon when a high pressure is applied to a solution with a high concentration.

On the other hand reverse osmosis filter to a concentration that gave a high pressure side and the dark solution is to use a reverse osmosis Anti-osmosis water is moved toward the concentration of concentrated solution.

The reverse osmosis filter uses an osmotic membrane in the form of a film with a pore size of about $0.0001 \mu \mathrm{m}$, and it is widely applied to household water purifiers. In addition, it has an 
ability to remove more than $90 \%$ of ionic substances including organic and inorganic pollutants, bacteria, and heavy metals. However, since minerals that are beneficial to the human body are removed and the amount of purified water is low due to low permeability, a high pressure pump is required from the outside, and a large amount of water must be discarded to obtain a small amount of purified water. Therefore, in order to improve the low transmittance, it is necessary to develop a new concept of a water purification membrane. On the other hand, another problem of the drinking supply device using a separation membrane is a fouling phenomenon in which a solid material is stuck in the micropores of the separation membrane to block the pores. Due to this phenomenon, as time passes, the permeated flow rate decreases, and secondary contamination of bacteria may occur. Due to this phenomenon, as time passes, the permeated flow rate decreases, and secondary contamination of bacteria may occur.

This with the flow rate of the more permeable over time, because of the reduced and may cause secondary pollution problems in bacteria.

In order to solve this problem, a technology for improving water purification efficiency by increasing the area of a backwashing water supply device or a membrane filter has been developed [7][8][9][10][11].

The problem with the water purifier mentioned so far can be summarized as follows. 1) Unnecessary energy is consumed because the cooling system continuously operates to maintain the water in the cooling tank at a constant cold water temperature. 1) Unnecessary energy is consumed because the cooling system continuously operates to maintain the water in the cooling tank at a constant cold water temperature.

1) it has become unnecessary energy consumption so that the cooling system to operate continuously in order to maintain the cooling water in the cylinder at a constant cold temperature.

In order to cool the water stored in the cooling vessel, a cooling coil is installed outside the cooling vessel and a pipe through which purified water passes is inserted into the cooling vessel to cool purified water. 2) The water remaining in the water purification pipe inserted inside the cooling tank is cooled, but it is difficult to maintain the required constant temperature of $12{ }^{\circ} \mathrm{Cbecause}$ the cooling water temperature rises due to the newly introduced purified water and the cooling performance of the discharged purified water decreases. 3) If cold water is continuously discharged, the amount of water used increases by more than a certain amount and the temperature of the water discharged increases, resulting in a decrease in cooling performance. 4) The life of the compressor can be shortened due to the frequent mechanical operation of the compressor.

In this study, by improving the existing instantaneous cooling system, the cooling coil is directly in contact with the cold water pipe so that heat is directly transferred by the direct heat generated between the cooling coil and the cold water pipe, thereby contributing to the energy saving that is a problem.

\section{Experiment}

\subsection{Experimental set-up}

A general device for testing the performance of a water purifier with an instant cooling system is equipped with a water filter to purify the water received, and the hot water is supplied to drinking water through a heater, and the cooling system consists of a compressor, a condenser and an expansion valve, and supplies cold water. 
Moment typical apparatus for testing the water purifier performance applying the cooling system is equipped with a water filter to purify the obtained water and hot water is supplied to the drinking water through the heating cooling system was composed of a compressor, a condenser and an expansion valve, cold water supply

A general device for testing the performance of a water purifier with an instant cooling system is equipped with a water filter to purify the water received, and the hot water is supplied to drinking water through a heater, and the cooling system consists of a compressor, a condenser and an expansion valve, and supplies cold water. A general device for testing the performance of a water purifier with an instant cooling system is equipped with a water filter to purify the water received, and the hot water is supplied to drinking water through a heater, and the cooling system consists of a compressor, a condenser and an expansion valve, and supplies cold water.

Moment typical apparatus for testing the water purifier performance applying the cooling system is equipped with a water filter to purify the obtained water and hot water is supplied to the drinking water through the heating cooling system was composed of a compressor, a condenser and an expansion valve, cold water supply

For this purpose, a cooling coil is installed in the insulated cooling system so that drinking water supply stainless steel pipe and hot water can be directly made. The diameter of the copper coil and the stainless-steel pipe (SUS 304) for supplying cold water is $10 \mathrm{~mm}$ each, and the height is $70 \mathrm{~cm}$. A $200 \mathrm{~W}$ compact compressor for cooling coil and raw cold water was applied, and a $600 \mathrm{~W}$ heater for hot water was applied to the water purifier system. A schematic diagram of the entire water purifier system and cooling system is shown in Figure A schematic diagram of the entire water purifier system and cooling system is shown in [Figure 1].Schematic view of the entire water purifier system and cooling system is shown in [Figure 1].

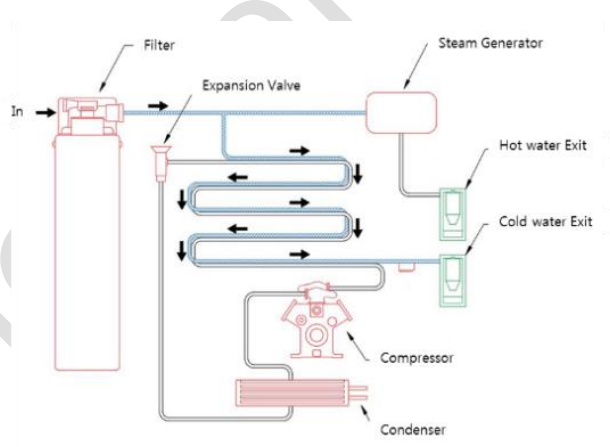

Figure 1. Schematic diagram for a water purification system

\subsection{Experimental method}

First, in order to secure the cold water discharge performance, the cold water temperature of the cooling system was set to $8^{\circ} \mathrm{C}$, and after stabilizing for 10 hours, the cold water started to be discharged. First, in order to secure the cold water discharge performance, the cold water temperature of the cooling system was set to $8^{\circ} \mathrm{C}$, and after stabilizing for 10 hours, the cold water started to be discharged.First, after setting the water temperature of the cooling system to stabilize and then $8{ }^{\circ} \mathrm{C}$ for 10 hours in order to secure the cold water outflow pipe performance started to the cold water outflow pipe.

The change in the temperature of the cold water was measured as time passed while increasing the amount of cold water outlet water from 1 to 10 liters. Second, in order to 
measure the hot water performance, the hot water temperature was set to $85^{\circ} \mathrm{C}$, stabilized for 8 hours, and then the heater operating time was measured while increasing the amount of hot water from 1 to 10 liters. Third, in order to measure standby power consumption, the temperature of the cooling system was set to $12^{\circ} \mathrm{C}$ and only the cooler was operated. After that, after being stabilized for 8 hours, the cooler was stopped and the standby power consumption $[\mathrm{Wh}]$ was measured.

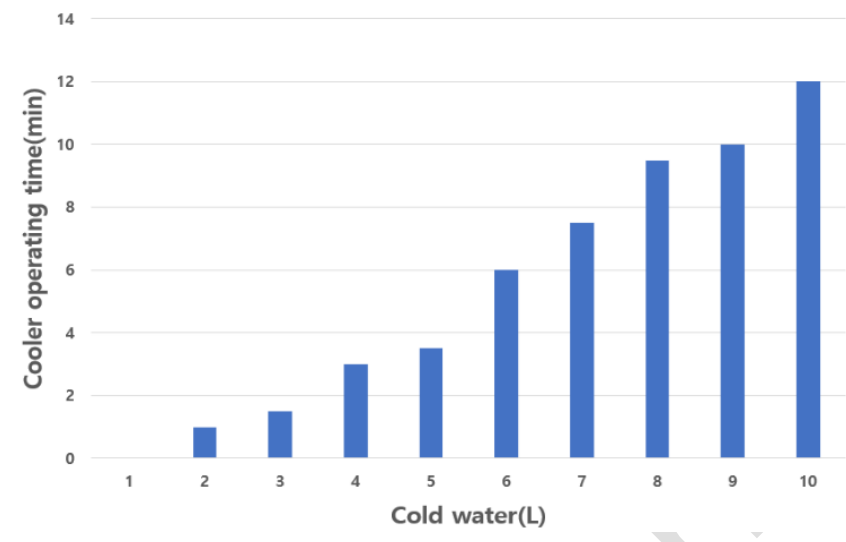

Figure 2. Cold water quantity vs. cold water temp

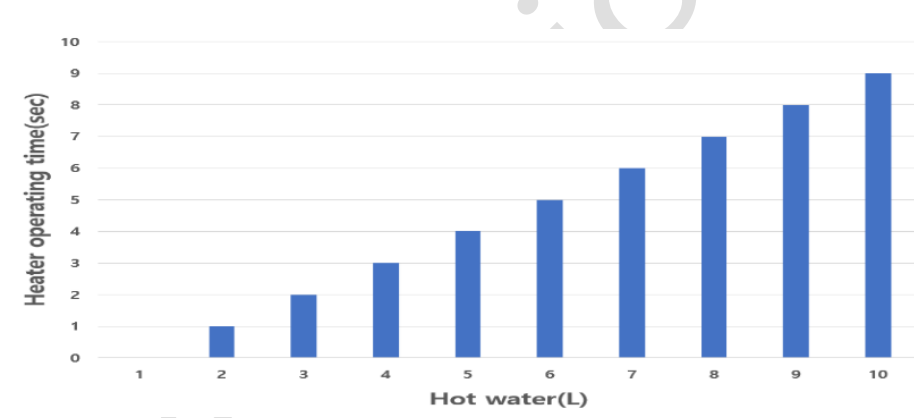

Figure 3. Cooler operating time for maintaining cold water temp

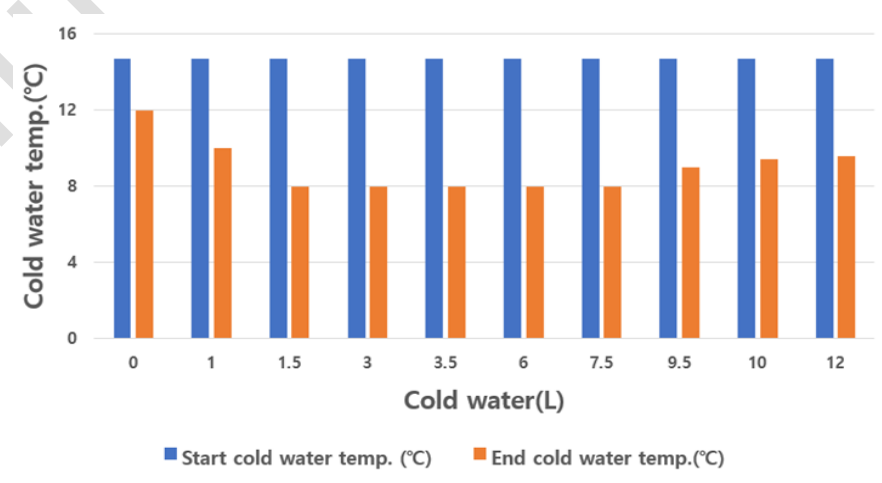

Figure 4. Hot water quantity vs. hot water temp. 


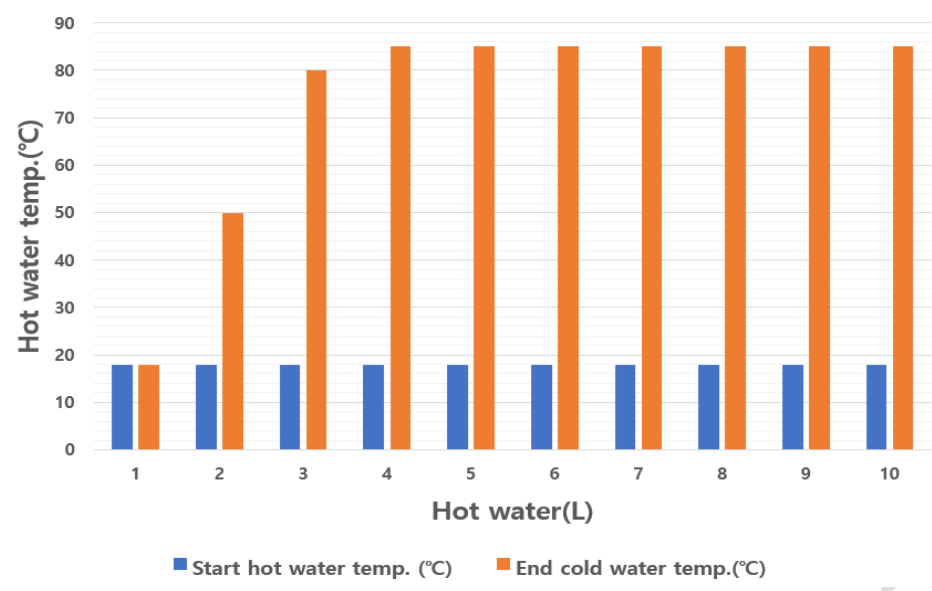

Figure 5. Heater operating time for maintaining lot water quantity (Liter)

\section{Results}

As a result of the cold water discharge performance test, the starting water temperature was $14.7^{\circ} \mathrm{C}$, and the cooling system was maintained at $8^{\circ} \mathrm{C}$ until 7.5 minutes after operation, and the water discharge temperature gradually increased from 9.5 minutes. As a result of the cold water discharge performance test, the starting water temperature was $14.7^{\circ} \mathrm{C}$, and the cooling system was maintained at $8^{\circ} \mathrm{C}$ until 7.5 minutes after operation, and the water discharge temperature gradually increased from 9.5 minutes.

Cold water was the heading performance of the test results, starting outflow water temperature is the cooling system to maintain a $14.7^{\circ} \mathrm{C} 8^{\circ} \mathrm{C}$ to 7.5 minutes after the operation elapsed time is increased by the outflow water temperature gradually From 9.5 minutes.

At 10 liters of cold water discharge, the cold water temperature was $9.6^{\circ} \mathrm{C}$ and the required time was 13 minutes See [Figure 2] At 10 liters of cold water discharge, the cold water temperature was $9.6^{\circ} \mathrm{C}$ and the required time was 13 minutes liters of cold water in the cold water chulsuryang temperature was $9.6^{\circ} \mathrm{C}$ time took 13 minutes [Figure 3] shows the amount of cold water discharged was based on 10 liters, and the operating time of the cooling system was shown as the amount of cold water discharged increased. In 4, the amount of cold water discharged was based on 10 liters, and the operating time of the cooling system was shown as the amount of cold water discharged increased.

The cold water 4 is chulsuryang was based on a 10-liter, it is shown the operating time of the cooling system resulting from the cold water chulsuryang increases.

The operating time per liter took $1 \mathrm{~min}$ on average.

[Figure 4], the hot water temperature of the water purifier is generally 85 degrees, and the hot water temperature is set to $85^{\circ} \mathrm{C}$ in order to measure the performance of the hot water. In 5 , the hot water temperature of the water purifier is generally 85 degrees, and the hot water temperature is set to $85^{\circ} \mathrm{C}$ in order to measure the performance of the hot water.

In general, the hot water temperature of the water purifier 85 the road heading has set the hot water temperature to measure the hot water performance with $85^{\circ} \mathrm{C}$ obtained temperature for starting with $14.7^{\circ} \mathrm{C}$ the temperature is raised from 2 liters in $3180^{\circ} \mathrm{C}, 4$ riteo. The target hot water temperature was reached at $85^{\circ} \mathrm{C}$.

The target hot water temperature was reached at $85^{\circ} \mathrm{C}$.

[Figure 5] shows the correlation of the elapsed time of the heater according to the amount of hot water. It took only 9 seconds to reach 10 liters of hot water at $85^{\circ} \mathrm{C}$. 


\section{Conclusion}

This study applied an instant cooling system that operates only when drinking water, unlike the existing method of cooling purified water. This study applied an instant cooling system that operates only when drinking water, unlike the existing method of cooling purified water.

This study is the traditional way of cooling the purified water was applied to the instantaneous cooling system that works only when drinking differently.

To cool the water in the cooling tank, a cooling coil (refrigerant pipe) was installed on the outside of the cooling tank, and a pipe through which purified water passed was inserted. An instant cooling system was developed to increase the amount of cold water output at $12^{\circ} \mathrm{C}$ more than three times from 3 liters to 10 liters. Unlike the existing instant cooling system, the cooling coil with refrigerant is directly in contact with the cold water pipe to maintain the existing cold water bottle by reducing standby power by $50 \%$ from $100 \mathrm{~W}$ to $50 \mathrm{~W}$, contributing to energy saving. By removing the unnecessary cold water bottle, the concern about the occurrence of bacteria has been eliminated, making it easier to manage the water bottle.

By removing the unnecessary cold water bottle, the concern about the occurrence of bacteria has been eliminated, making it easier to manage the water bottle.

By removing unnecessary water bottle by removing concerns about germs it was easily generated through the constant management.

In addition, unlike existing water purification systems that have unnecessary cooling water tanks attached to the outside, the system is easy to simplify, making it possible to manufacture a small size water purifier. In addition, unlike existing water purification systems that have unnecessary cooling water tanks attached to the outside, the system is easy to simplify, making it possible to manufacture a small water purifier.

In addition, unlike the conventional water purification system to mount an unnecessary cooling water tube to the outside by the simplification of the system it made it possible to readily manufacture a small water purifier.

In addition, the conduction-type instantaneous cooling system developed through this study was able to secure a second class of power consumption efficiency.

\section{References}

[1] Y. S. Jeon, H. H. Park, J. S. Lee, "Analytical study on the performance characteristics of an instant cooling type water purifier with the design parameters of the cooling tank," Korean J. of Air-conditioning and Refrigeration Engineering, vol.24, no.7, pp.545-552, (2012)

[2] K. J. Park, Y. H. Lee, D. S. Jung, and K. K. Kim, "Performance of G430A on refrigeration system of domestic water purifiers," Korean J. of Air-conditioning and Refrigeration Engineering, vol.21, no.2, pp.109-117, (2009)

[3] J. W. Jung, C. W. Jung, S. S. Kim, and Y. T. Kang, "Performance comparison between the conventional air cooled type condenser and the water cooled type condenser using drain water of the reverse osmosis type cold and hot water purifier," Proceedings of SAREK Summer Annual Conference, pp.1165-1170, Sept. 7-10, (2015)

[4] D. W. Lee, and C. B. Kim, "Characteristic analysis of the cooling system using ice slurry type heat storage system,” J. of Energy Engineering, vol.20, no.1, pp.30-35, (2011)

[5] F. P. Incropera and D. P. Dewitt, "Heat and mass transfer," John Wiley Publishers, (2017)

[6] K. B. Kim, "A design compact size direct feed water purifier through the redesign of cooling module part," Thesis, Pusan National University, Korea, (2015) 
[7] J. H. Lee, "A study on factors of agitator of cooling module in direct water stream type of water purifier that influence continuous cooled water outlet temperature," Thesis, Pusan National University, Korea, (2014)

[8] J. S. Kim, "Experimental study on cooling performance of instant cooling unit for drinking water," Thesis, Seoul National University of Science and Technology, (2017)

[9] Yunus A. Cengel and Michael A. Boles, "Thermodynamics," McGraw Hill, (2016)

[10] D. H. Kim and S. H. Lee, "Evolution of water purifier technology due to social environment changes," Journal of Korean Society for Environment Technology, vol.2, no.5, pp.240-248, (2020)

[11] K. H. Ko and Y. W. Hwang, "Life cycle water footprint analysis of an water purifier product," Korea Society for life cycle assessment, vol.19, no.1, pp.41-43, (2018) 
Development of Direct Water Purification System

This page is empty by intention. 